\title{
Molecular characterization of a cluster of at least two glucosyltransferase genes in Streptococcus salivarius ATCC 25975
}

\author{
Philip M. Giffard, Christine L. Simpson, Carolyn P. Milward and Nicholas A. Jacques* \\ Institute of Dental Research, United Dental Hospital of Sydney, Surry Hills, New South Wales 2010, Australia
}

(Received 5 April 1991; revised 30 July 1991; accepted 7 August 1991)

\begin{abstract}
The oral micro-organism Streptococcus salivarius ATCC 25975 synthesizes extracellular glucosyltransferases (GTFs) which polymerize the glucose moiety of sucrose into glucan polymers. Two separate genes encoding the activities of a GTF-I (a GTF that synthesizes an insoluble product) and a GTF-S (a GTF that synthesizes soluble product) were cloned into bacteriophage $\lambda$ L47.1. The inserts in the $\lambda$-clones were characterized by restriction mapping and Southern hybridization and were found to overlap, implying that the two genes lay very close to one another on the $S$. salivarius chromosome. Both genes were subcloned into phagemid vector pIBI30 where they were expressed at a high level. The GTF-I-encoding gene was named $g t f J$ and the GTF-S-encoding gene, $g t f K$. Nucleotide sequencing showed that $g t f J$ and most probably $g t f K$ were closely related to the $g t f$ genes of the mutans streptococci. Sequence alignment also indicated that $g t f K$ lay very close to and downstream from $g t f J$, and that both were transcribed in the same direction.
\end{abstract}

\section{Introduction}

Streptococcus salivarius is the most abundant streptococcal species in the human oral cavity, becoming established $24 \mathrm{~h}$ after birth. Animal experiments have led to many strains being classified in the 'high' cariogenic category along with the mutans streptococci (Krasse \& Carlsson, 1970; Drucker et al., 1984) and like the mutans streptococci these strains of $S$. salivarius produce extracellular glucosyltransferases (GTFs) that polymerize the glucose moiety of sucrose into glucan polymers. The GTFs secreted by $S$. salivarius are likely to be active not only in the saliva but also in the acquired pellicle formed on the tooth surface (Rölla et al., 1983). Thus, in the presence of sucrose, GTFs secreted by $S$. salivarius may aid in the initial attachment or entrapment of their own as well as other oral species to a newly erupted tooth surface or to a tooth surface following prophylaxis.

Biochemical studies have demonstrated that the GTFs synthesized by oral streptococci can be divided into two broad categories - those that synthesize an $\alpha-(1 \rightarrow 3)$ linked insoluble polymer (GTF-I) and those that synthe-

Abbreviation: GTF, glucosyltransferase.

The nucleotide sequence data reported in this paper have been submitted to GenBank and have been assigned the accession numbers M64111 (gtfJ; Fig. 4) and M64112 (gtfK; Fig. 6). size an $\alpha-(1 \rightarrow 6)$-linked soluble polymer (GTF-S). These enzymes work together in situ to synthesize glucans of mixed $\alpha-(1 \rightarrow 3)$ - and $\alpha-(1 \rightarrow 6)$-linkages (Walker \& Jacques, 1987). Recently, a number of genes encoding GTF enzymes have been isolated. Of these the $g t f I$ and $g t f S$ genes of $S$. downei $\mathrm{MFe} 28$ and the $g t f B, g t f C$ and $g t f D$ genes of $S$. mutans strain GS5 (which encode a GTF-I and two GTF-S activities respectively) have been sequenced (Ferretti et al., 1987; Shiroza et al., 1987; Ueda et al., 1988; Gilmore et al. 1990; Honda et al., 1990) and it has been found that all these genes are closely related to each other. In the case of $S$. mutans GS5 the $g t f B$ and $g t f C$ genes, but not the $g t f D$ gene, lie in close proximity to one another (Perry \& Kuramitsu, 1990).

We have recently reported the isolation of GTF-I- and GTF-S-positive clones from S. salivarius ATCC 25975 DNA (Pitty et al., 1989). $S$. salivarius is not a member of the mutans group so it was of interest to determine if these genes were related to those from within that group. Also, as expression of GTF-I and GTF-S activity in S. salivarius ATCC 25975 is co-ordinately regulated, possibly at the level of protein translocation (Pitty \& Jacques, 1987), it was of interest to determine if the two cloned genes were closely linked on the $S$. salivarius chromosome. In this communication, we not only demonstrate this is indeed the case, but also show that the gene encoding the GTF-I and probably the gene encoding the GTF-S of $S$. salivarius ATCC 25975 are 
(a)

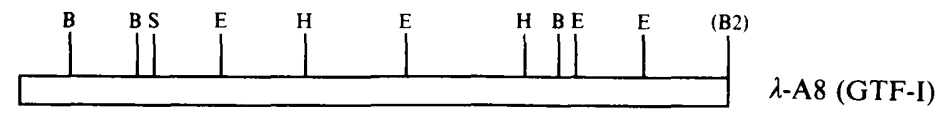

(b)

$\lambda$-A33 (GTF-S)

(c)

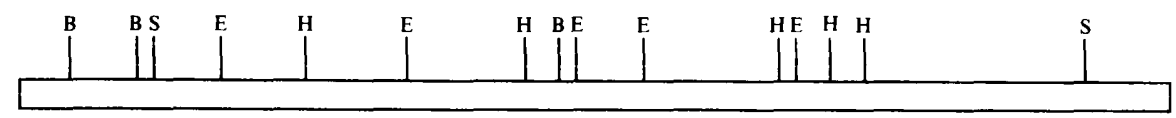

(d)

(e)

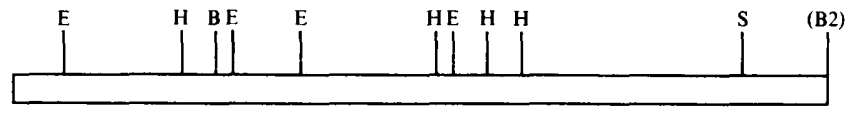

GTF-S subclone (pGS201)

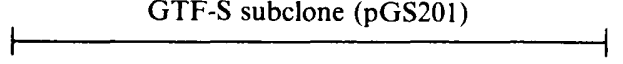

)

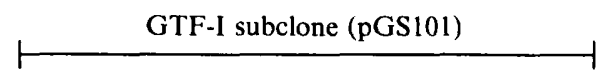

)

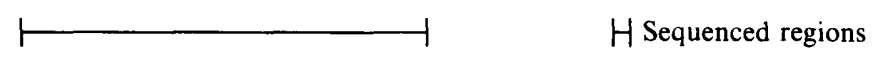

(f)

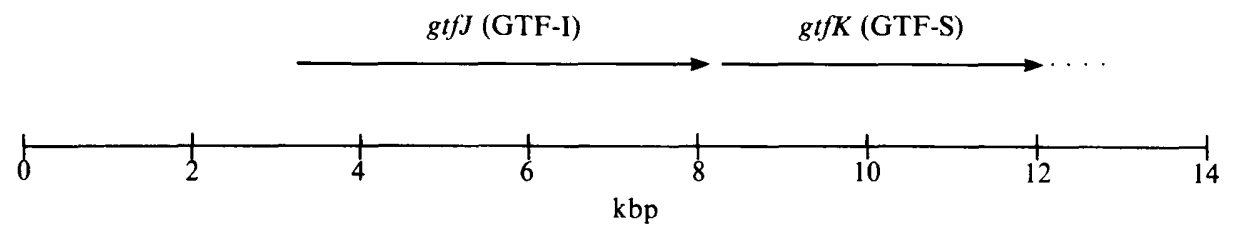

Fig. 1. Contiguous locus $(c)$ formed from the merging of the restriction maps of the inserts in $\lambda A-8(a)$ and $\lambda A-33(b)$. The area of apparent overlap is indicated by the double line. Also shown are the 1.9 and $0.9 \mathrm{kbp} \mathrm{EcoRI} \mathrm{fragments} \mathrm{purified} \mathrm{from} \lambda \mathrm{A}-8$ and used to probe $E c o$ RI digests of $\lambda \mathrm{A}-8$ and $\lambda \mathrm{A}-33$ (see text), the fragments $(d)$ cloned into pIBI30 to yield pGS101 (GTF-I positive clone) and pGS201 (GTF-S positive clone), the regions subject to nucleotide sequence analysis $(e)$ and the extent of the open-reading frames for $g t f J$ and $g t f K$ where the arrows indicate the direction of transcription of the two genes $(f)$. B, BglII ; B2, Bam HI; E, Eco RI; H, HindIII; S, SacI. No ApaI sites were found. Note that the bracketed BamHI sites are derived from the ligation of a SauIIIA fragment to a BamHI fragment. That in $\lambda \mathrm{A}-8$ is known not to exist on the chromosome.

closely related to the gtf genes from the mutans streptococci.

\section{Methods}

Reagents, chemicals and enzymes. Mutanolysin was kindly supplied by Dr K. Yokogawa, Dainippon Pharmaceutical Co., Osaka, Japan. $\left[\alpha-{ }^{32} \mathrm{P}\right] \mathrm{dCTP}$ and $\left[\alpha-{ }^{35} \mathrm{~S}\right] \mathrm{dATP}-\alpha \mathrm{S}$ were purchased from NEN, and restriction enzymes, exonucleases, T4 DNA ligase and salmon sperm DNA from Boehringer and/or Pharmacia. Custom oligonucleotides were obtained from Dr A. Weiss in the Department of Biochemistry at the University of Sydney, Australia. Tween 80, dextran T10, dextran sulphate, Triton $\mathrm{X}-100$ and triphenyltetrazolium chloride were obtained from Sigma and skim milk from Oxoid. All other reagents were of analytical reagent grade or of the highest grade available.

Bacterial strains, bacteriophage, plasmids and growth conditions. All strains and genetic constructs, excluding sequencing subclones, are listed in Table 1. Escherichia coli was grown in LB medium (Maniatis et al., 1982). Bacteriophage derivatives were grown either as 1 litre liquid
Table 1. Strains and plasmids

\begin{tabular}{ll}
\hline \hline Strain or plasmid & \multicolumn{1}{c}{ Source or reference } \\
\hline S. salivarius & \\
ATCC 25975 & ATCC, Bethesda, USA (Hamilton, 1967) \\
E. coli & \\
LE392 & Murray et al. (1977) \\
JM105 & Yannisch-Perron et al. (1985) \\
NM522 & Gough \& Murray (1983) \\
Bacteriophage & \\
$\lambda$ L47.1 & Loenen \& Brammar (1980) \\
$\lambda$ A-8 & Encodes GTF-I (Pitty et al., 1989) \\
$\lambda$ A-33 & Encodes GTF-S (Pitty et al., 1989) \\
M13K07 & Purchased from IBI \\
Plasmid & \\
pIBI30 & Purchased from IBI \\
pGS101 & pIBI30 with GTF-I encoding SacI-BamHI \\
pGS201 & fragment (this study) \\
& pIBI30 with GTF-S encoding BglII-BamHI \\
\hline \hline
\end{tabular}


(a)
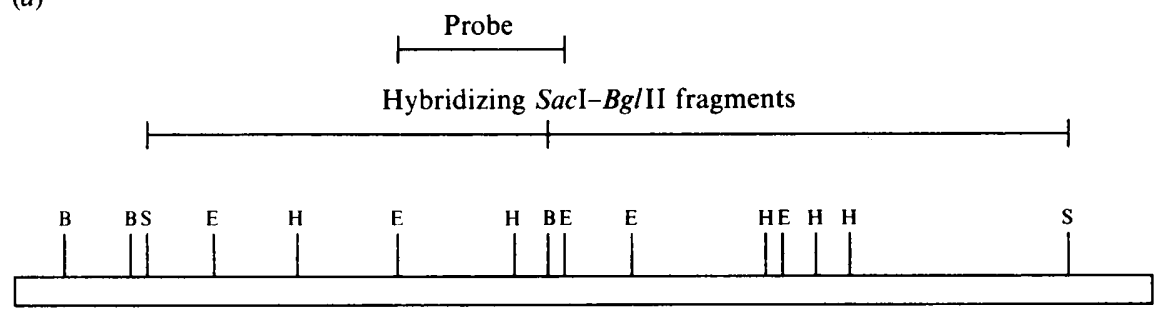

$1 \mathrm{kbp}$

(b)

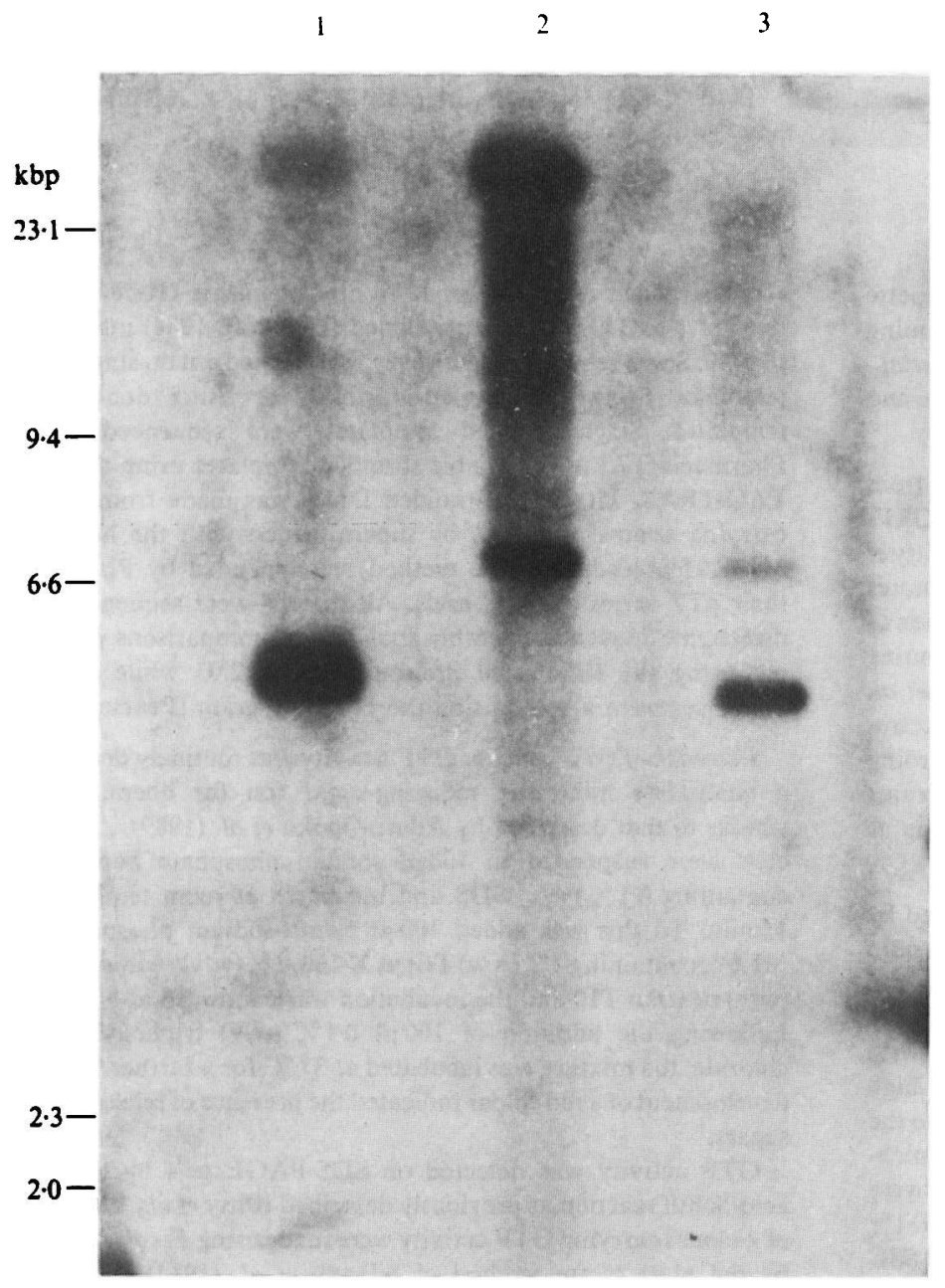

Fig. 2. Southern hybridization analysis of the $S$. salivarius chromosome. (a) The $1.9 \mathrm{kbp}$ EcoRI fragment used to probe the $S a c I-B g l$ II double digests of $\lambda \mathrm{A}-8, \lambda \mathrm{A}-33$ and $S$. salivarius chromosomal DNA. (b) SacI-BglII double digests of $\lambda \mathrm{A}-8$ (lane 1); $\lambda \mathrm{A}-33$ (lane 2); $S$. salivarius chromosomal DNA (lane 3).

lysates or as plate lysates according to the method of Silhavy et al. (1984) using E. coli strain LE392 as the host. Plasmids were propagated in either $E$. coli strain JM105 or strain NM522.

Purification of chromosomal DNA from $S$. salivarius. S. salivarius ATCC 25975 was grown in $400 \mathrm{ml}$ of Todd-Hewitt broth at $37^{\circ} \mathrm{C}$ until late exponential phase $\left(\mathrm{OD}_{600} 0.6-0.8\right)$ at which point glycine was added to $20 \%(\mathrm{w} / \mathrm{v})$ and incubation continued for a further $1 \mathrm{~h}$. The cells were harvested by centrifugation $\left(10000 \mathrm{~g}, 4^{\circ} \mathrm{C}, 10 \mathrm{~min}\right)$, washed

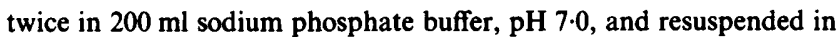
$30 \mathrm{ml}$ of the same buffer. Following the addition of $5 \mathrm{~mm}$-EDTA, $0.5 \%$ (v/v) Triton X-100,100 $\mathrm{gg}$ mutanolysin $\mathrm{ml}^{-1}$ and $50 \mu \mathrm{g}$ proteinase $\mathrm{K} \mathrm{ml}{ }^{-1}$, the cells were incubated for $2 \mathrm{~h}$ at $37^{\circ} \mathrm{C}$ followed by $1 \mathrm{~h}$ at $50{ }^{\circ} \mathrm{C}$, at which time $0.2 \%$ (w/v) SDS was added. The resulting translucent, viscous solution was extracted four times with equal volumes of Tris/HCl-saturated phenol $(\mathrm{pH} \mathrm{8.0)}$ and once with an equal volume of chloroform/isoamyl alcohol $(24: 1, \mathrm{v} / \mathrm{v})$. Following the precipitation of DNA by the addition of $0.1 \mathrm{vol} .3 \mathrm{M}$-sodium acetate 


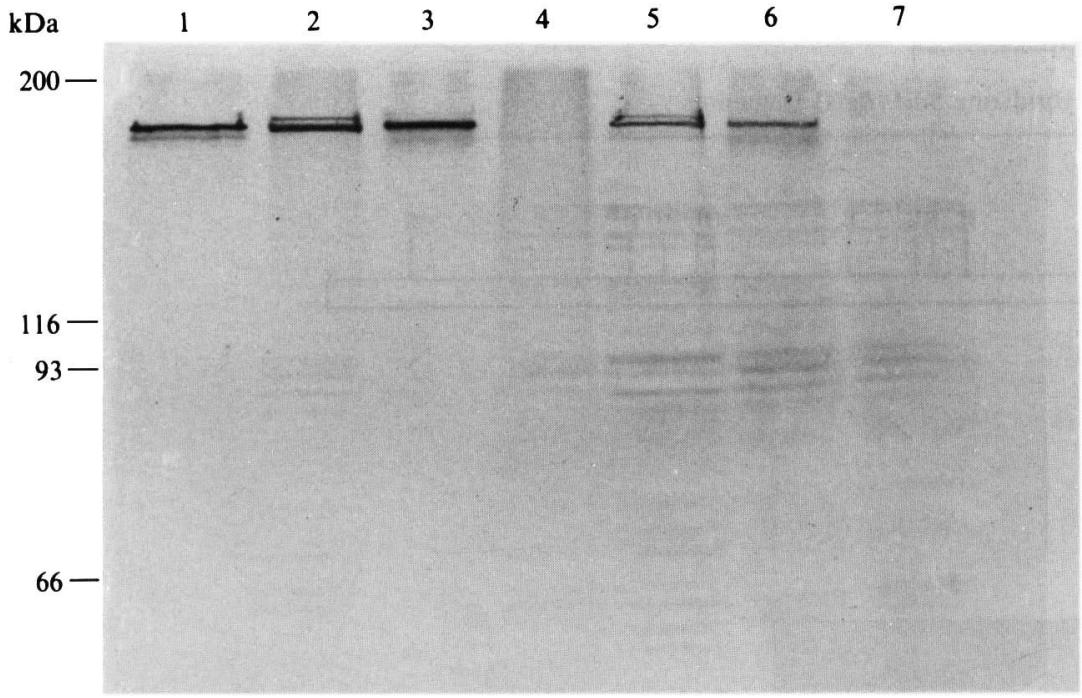

Fig. 3. Periodic acid/Schiff staining for GTF activity following SDS-PAGE analysis of cloned gene products. Culture fluid of $S$. salivarius grown in the presence of $0.05 \mu \mathrm{l}$ Tween $80 \mathrm{ml}^{-1}$ (lane 1); $\lambda \mathrm{A}-33$ lysate (lane 2 ); $\lambda A-8$ lysate (lane 3 ); $\lambda$ L47.1 lysate (lane 4); E. coli NM522 with pGS201 (lane 5); E. coli NM522 with pGS101 (lane 6); E. coli NM522 devoid of plasmid (lane 7). and 2 vols ethanol, the DNA was spooled on to a Pasteur pipette and then dissolved in $5 \mathrm{ml} 50 \mathrm{~mm}-\mathrm{Tris} / \mathrm{HCl}, \mathrm{pH} 7.5$, containing $1 \mathrm{~mm}$-EDTA and $200 \mu \mathrm{g}$ heat-treated RNAase $\mathrm{ml}^{-1}$ (Maniatis et al., 1982). The DNA was then reprecipitated as described above and redissolved in $5 \mathrm{ml} 50 \mathrm{~mm}$-Tris/ $\mathrm{HCl}, \mathrm{pH} \mathrm{7.5,1} \mathrm{mm}$-EDTA.

DNA manipulations. The construction of a gene bank from $S$. salivarius DNA and the isolation and characterization of the GTFpositive clones $\lambda A-8$ and $\lambda A-33$ have been described previously (Pitty $e t$ al., 1989). Bacteriophage $\lambda$ DNA was purified from 1 litre liquid lysates according to the method of Silhavy et al. (1984). Both the purification of plasmids from $E$. coli by the alkaline lysis method and the routine manipulation of DNA in vitro were as described by Maniatis $e t$ al. (1982). DNA fragments were purified from agarose gels by electrophoresis on to DE81 ion-exchange paper (Whatman) according to Dretzen et al. (1981). Transformation of $E$. coli strains was by electroporation using a Bio-Rad Gene Pulser apparatus as recommended by the manufacturer.

Southern hybridizations. These were done essentially as outlined by Silhavy et al. (1984). DNA was transferred from $0.7 \%$ agarose gels onto Hybond $\mathrm{N}$ nylon membrane (Amersham). Prehybridizations $(4 \mathrm{~h})$ and hybridizations $(16 \mathrm{~h})$ were carried out at $65^{\circ} \mathrm{C}$ in $1.5 \times$ SSPE buffer (Maniatis et al., 1982) containing 0.5\% (w/v) skim milk, 0.5\% (w/v) SDS and $250 \mu \mathrm{g}$ denatured salmon sperm DNA ml-1. When high sensitivity was required $10 \%(\mathrm{w} / \mathrm{v})$ dextran sulphate was added to the hybridization mix. Probes were labelled with [ $\left.{ }^{32} \mathrm{P}\right] \mathrm{dCTP}$ by nicktranslation using a kit supplied by BRL. Stringency washes were performed with $2 \times \mathrm{SSC}$ buffer (Maniatis et al., 1982) containing $0.1 \%$ (w/v) SDS at room temperature (two washes) followed by $0 \cdot 1 \times$ SSC buffer (Maniatis et al., 1982) containing $0 \cdot 1 \%(\mathrm{w} / \mathrm{v}) \mathrm{SDS}$ at $65^{\circ} \mathrm{C}$ (four washes).

DNA sequence determination. All sequencing was done by the dideoxy method (Sanger, 1977). The majority of this sequencing was done on single-stranded templates made from exonuclease-III-deleted derivatives of 1 to $3 \mathrm{kbp}$ fragments cloned (Henikoff, 1984) into phagemid pIBI30. Some sequencing, however, was carried out on single-stranded templates using custom-made primers or using double-stranded templates. Single-stranded templates were sequenced using the Pharmacia T7 kit and double-stranded templates using the Promega TAQ-TRAK kit. Single-stranded DNA was made from phagemidcarrying strains of $E$. coli by superinfection with the helper phage M13K07 according to the method recommended by Pharmacia for their pTZ series of phagemids. All regions were sequenced in both directions. Sequence assembly analysis and comparisons were carried out using the IBI-Pustell program version 2.03 while amino acid sequences were aligned using the FastP program (Pearson, 1990).

Detection of GTF activity. GTF activity was routinely detected using a qualitative microtitre reducing-sugar test for liberated fructose similar to that described by Aduse-Opoku et al. (1989). A loopfull of cells were suspended in $100 \mu \mathrm{l}$ sodium phosphate buffer, pH 6.5, containing $0.1 \%(w / v)$ SDS and incubated at room temperature for $15 \mathrm{~min}$. To this was added $100 \mu \mathrm{l} 50 \mathrm{~mm}$-sodium phosphate buffer, pH 6.5 , containing $1 \%(\mathrm{w} / \mathrm{v})$ Triton $\mathrm{X}-100,1 \%(\mathrm{w} / \mathrm{v})$ sucrose and $0.04 \%$ (w/v) dextran T10 and the incubation was continued at $37^{\circ} \mathrm{C}$ for $1 \mathrm{~h}$. Following the addition of $100 \mu \mathrm{l} 0.1 \%(\mathrm{w} / \mathrm{v})$ triphenyltetrazolium chloride, the mixture was incubated at $37^{\circ} \mathrm{C}$ for a further $15 \mathrm{~min}$. The development of a red colour indicated the presence of released reducing sugars.

GTF activity was detected on SDS-PAGE gels by the periodic acid/Schiff reaction as previously described (Pitty et al., 1989). Lysates of $\lambda$-clones carrying GTF activity were made using $E$. coli strain LE392 by the plate lysate method of Silhavy et al. (1984). GTF activity encoded by the phagemids was released from $E$. coli cells by permeabilizing $1 \mathrm{ml}$ of a stationary phase culture. This was achieved by vortexing the cells in the presence of $50 \mu \mathrm{l} 0.1 \%$ (w/v) SDS and $100 \mu \mathrm{l}$ chloroform for $20 \mathrm{~s}$.

Fig. 4. Nucleotide sequence of the $g t f J$ gene and the deduced amino acid sequence of its product. The location of the sequenced fragment is indicated in the text. The putative ribosome binding site (RBS) is shown at nucleotide 13. The regions similar to the 'A' portion of the 'AC' repeat consensus sequence are underlined with a single line while the ' $D$ ' repeats are underlined with asterisks. Also shown is the putative 5 '-P end of the $g t f K$ gene beginning at nucleotide 4790 . This nucleotide sequence has been submitted to GenBank and is listed under accession number M64111. 


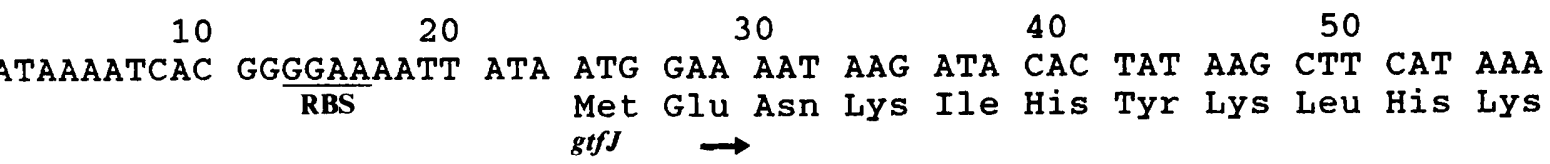
$\begin{array}{lllll}60 & 70 & 80 & 90 & 100\end{array}$ GTT AAG AAG CAA TGG GTT ACA ATT GCA GTT GCT TCT GTA GCA CTT GCT ACT Val Lys Lys Gln Trp Val Thr Ile Ala Val Ala Ser Val Ala Leu Ala Thr $\begin{array}{lllll}110 & 120 & 130 & 140 & 150\end{array}$

GTC TTG GGA GGA TTG TCT GTA ACA ACA TCT TCA GTT TCA GCG GAT GAA ACT Val Leu Gly Gly Leu Ser Val Thr Thr ser ser Val Ser Ala Asp Glu Thr
160 170
180
190 200

CAA GAT AAG ACA GTA ACT CAA TCA AAT TCA GGT ACA ACA GCT TCT TTA GTT Gln Asp Lys Thr Val Thr Gln Ser Asn Ser Gly Thr Thr Ala Ser Leu Val

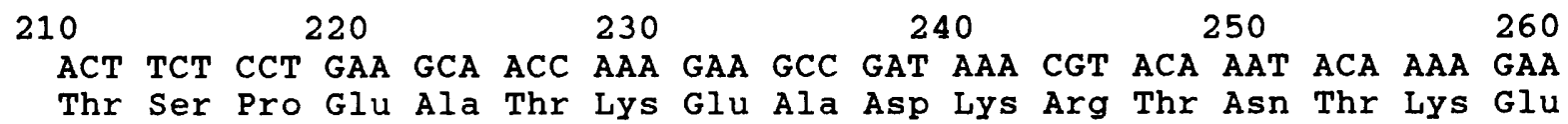

$$
270 \quad 280 \quad 290 \quad 300 \quad 310
$$

GCA GAT GTT TTA ACA CCT GCT AAA GAA ACA AAT GCT GTA GAA ACA GCG ACT Ala Asp Val Leu Thr Pro Ala Lys Glu Thr Asn Ala Val Glu Thr Ala Thr

$$
320 \quad 330 \quad 340 \quad 350 \quad 360
$$

ACA ACG AAC ACA CAA GCA ACA GCT GAA GCA GCT ACA ACA GCA ACA ACA GCT Thr Thr Asn Thr Gln Ala Thr Ala Glu Ala Ala Thr Thr Ala Thr Thr Ala

$370 \quad 380 \quad 390 \quad 400 \quad 410$

GAT GTA GCA GTG GCA GCT GTT CCA AAT AAA GAA GCA GTT GTG ACA ACA GAT Asp Val Ala Val Ala Ala Val Pro Asn Lys Glu Ala Val Val Thr Thr Asp

$$
420 \quad 430 \quad 440 \quad 450 \quad 460
$$

GCA CCA GCT GTT ACA ACT GAA AAA GCA GAA GAA CAA CCA GCA ACA GTG AAG Ala Pro Ala Val Thr Thr Glu Lys Ala Glu Glu Gln Pro Ala Thr Val Lys

$$
\begin{array}{lllll}
470 & 480 & 490 & 500 & 510
\end{array}
$$

GCT GAA GTT GTT AAT ACA GAA GTT AAG GCG CCA GAA GCT GCT TTG AAA GAT Ala Glu Val Val Asn Thr Glu Val Lys Ala Pro Glu Ala Ala Leu Lys Asp

$520 \quad 530 \quad 540 \quad 550 \quad 560$

TCA GAA GTA GAA GCT GCG CTT TCC TTG AAA AAC ATC AAA AAC ATT GAT GGT Ser Glu Val Glu Ala Ala Leu Ser Leu Lys Asn Ile Lys Asn Ile Asp Gly

$$
570 \quad 580 \quad 590 \quad 600 \quad 610
$$

AAA TAT TAC TAT GTT AAT GAA GAT GGT TCA CAC AAA GAA AAC TTT GCC ATT Lys Tyr Tyr Tyr Val Asn Glu Asp Gly Ser His hys Glu Asn Phe Ala Ile

$620 \quad 630 \quad 640 \quad 650 \quad 660$

ACT GTA AAT GGT CAA TTG CTT TAC TTC GGT AAA GAT GGT GCT CTT ACA AGT Thr Val Asn Gly Gln Leu Leu Tyr Phe Gly Lys Asp Gly Ala Leu Thr Ser

$670 \quad 680 \quad 690 \quad 700 \quad 710$

TCA TCA ACA TAC TCT TTC ACA CCA GGA ACA ACA AAT ATT GTT GAT GGT TTC

Ser Ser Thr Tyr Ser phe Thr Pro Gly Thr Thr Asn Ile Val Asp Gly Phe

$\begin{array}{llllll}720 & 730 & 740 & 750 & 760 & 770\end{array}$

TCA ATA AAT AAC CGT GCC TAC GAT TCA TCT GAA GCT AGC TTT GAA TTG ATT Ser Ile Asn Asn Arg Ala Tyr Asp Ser Ser Glu Ala ser Phe Glu Leu Ile 

790

800

810

820

GAT GGT TAT TTG ACT GCA GAT AGC TGG TAC CGT CCA GCT TCT ATC ATC AAA Asp Gly Tyr Leu Thr Ala Asp Ser Trp Tyr Arg Pro Ala ser Ile Ile Lys GAT GGT $\begin{aligned} & 830 \\ & \text { GTA }\end{aligned}$ ACT TGG CAA GCA TCA ACT GCA GAA GAT $\begin{aligned} & 860 \\ & \text { TTC CGT CCA CTT TTG }\end{aligned}$ Asp Gly Val Thr Trp Gln Ala Ser Thr Ala Glu Asp Phe Arg Pro Leu Leu

$\begin{array}{lllll}880 & 890 & 900 & 910 & 920\end{array}$

ATG GCT TGG TGG CCA AAT GTA GAT ACA CAA GTT AAC TAC TTG AAC TAC ATG Met Ala Trp Trp pro Asn Val Asp Thr Gln Val Asn Tyr Leu Asn Tyr Met

$9300940 \quad 950 \quad 960 \quad 970$

TCT AAA GTA TTT AAC TTG GAT GCT AAA TAT TCA AGT ACA GAT AAG CAA GAA Ser Lys Val phe Asn Leu Asp Ala Lys Tyr ser Ser Thr Asp Lys Gln Glu $980 \quad 990 \quad 1000 \quad 1010 \quad 1020$

ACT TTG AAA GTT GCT GCT AAG GAC ATT CAA ATC AAG ATT GAG CAA AAG ATT Thr Leu Lys Val Ala Ala Lys Asp Ile Gln Ile Lys Ile Glu Gln Lys Ile

$1030 \quad 1040 \quad 1050 \quad 1060 \quad 1070$

CAG GCT GAA AAA TCA ACA CAA TGG TTG CGT GAA ACT ATC TCT GCC TTT GTT Gln Ala Glu Lys Ser Thr Gln Trp Leu Arg Glu Thr Ile Ser Ala Phe Val

$$
\begin{array}{lllll}
1080 & 1090 & 1100 & 1110 & 1120
\end{array}
$$

AAG ACA CAA CCA CAA TGG AAC AAA GAA ACT GAA AAC TAC TCT AAA GGT GGC Lys Thr Gln Pro Gln Trp Asn Lys Glu Thr Glu Asn Tyr Ser Lys Gly Gly

\begin{tabular}{rcccccc}
1130 & \multicolumn{1}{c}{1140} & 1150 & \multicolumn{2}{c}{1160} & & 1170 \\
GGC GAA & GAT CAC CTT & CAA & GGT GGT GCC & CTT CTT TAT GTG AAT GAT TCA CGT
\end{tabular}
Gly Glu Asp His Leu Gln Gly Gly Ala Leu Leu Tyr Val Asn Asp Ser Arg

$1180 \quad 1190 \quad 1200 \quad 1210 \quad 1220$

ACA CCA TGG GCG AAT TCT GAC TAT CGT CGT TTG AAC CGT ACA GCA ACT AAC Thr Pro Trp Ala Asn Ser Asp Tyr Arg Arg Leu Asn Arg Thr Ala Thr Asn

\begin{tabular}{|c|c|c|}
\hline & 30 \\
\hline
\end{tabular}

CAG ACT GGT ACA ATT GAT AAA TCA ATT CTT GAT GAG CAA TCA GAT CCA AAC Gln Thr Gly Thr Ile Asp Lys Ser Ile Leu Asp Glu Gln Ser Asp Pro Asn

$$
\begin{array}{lllll}
1290 & 1300 & 1310 & 1320 & 1330
\end{array}
$$

CAC ATG GGT GGT TTC GAC TTC TTG CTA GCT AAT GAC GTA GAT TTG TCA AAC His Met Gly Gly Phe Asp Phe Leu Leu Ala Asn Asp Val Asp Leu Ser Asn

$$
\begin{array}{lllll}
1340 & 1350 & 1360 & 1370 & 1380
\end{array}
$$

CCA GTT GTT CAA GCG GAA CAA TTG AAC CAA ATC CAC TAC CTT ATG AAC TGG Pro Val Val Gln Ala Glu Gln Leu Asn Gln Ile His Tyr Leu Met Asn Trp

$$
1390 \quad 1400 \quad 1410 \quad 1420 \quad 1430
$$

GGT TCA ATC GTT ATG GGT GAC AAG GAT GCT AAC TTC GAT GGT ATC CGT GTC Gly Ser Ile Val Met Gly Asp Lys Asp Ala Asn Phe Asp Gly Ile Arg Val

$$
\begin{array}{lllll}
1440 & 1450 & 1460 & 1470 & 1480
\end{array}
$$

GAC GCG GTA GAT AAT GTC GAT GCA GAC ATG CTT CAA CTC TAC ACA AAC TAC Asp Ala Val Asp Asn Val Asp Ala Asp Met Leu Gln Leu Tyr Thr Asn Tyr 
$1540 \quad 1550 \quad 1560 \quad 1570 \quad 1580$

ATC TCA GTC CTT GAA GCA TGG AGC CTT AAT GAC AAC CAC TAC AAT GAC AAG Ile Ser Val Leu Glu Ala Trp Ser Leu Asn Asp Asn His Tyr Asn Asp Lys $\begin{array}{lllll}1590 & 1600 & 1610 & 1620 & 1630\end{array}$

ACA GAT GGC GCT GCG CTT GCT ATG GAA AAC AAA CAA CGT TTG GCT CTC CTC Thr Asp Gly Ala Ala Leu Ala Met Glu Asn Lys Gln Arg Leu Ala Leu Leu

$\begin{array}{lllll}1640 & 1650 & 1660 & 1670 & 1680\end{array}$

TTC TCA TTG GCT AAA CCA ATC AAA GAA CGT ACA CCA GCT GTA AGT CCT TTG Phe Ser Leu Ala Lys pro Ile Lys Glu Arg Thr Pro Ala Val ser Pro Leu

$1690 \quad 1700 \quad 1710 \quad 1720 \quad 1730$

TAT AAC AAT ACT TTC AAC ACG ACA CAA CGT GAT GAA AAG ACT GAT TGG ATT

Tyr Asn Asn Thr Phe Asn Thr Thr Gln Arg Asp Glu Lys Thr Asp Trp Ile

$\begin{array}{llllll}1740 & 1750 & 1760 & 1770 & 1780 & 1790\end{array}$

AAC AAA GAT GGA AGC AAG GCC TAT AAC GAA GAC GGA ACA GTT AAA CAG TCT Asn Lys Asp Gly Ser Lys Ala Tyr Asn Glu Asp Gly Thr Val Lys Gln Ser

$\begin{array}{lllll}1800 & 1810 & 1820 & 1830 & 1840\end{array}$

ACA ATC GGT AAA TAT AAC GAG AAA TAC GGA GAT GCG TCA GGA AAT TAC GTC Thr Ile Gly Lys Tyr Asn Glu Lys Tyr Gly Asp Ala ser Gly Asn Tyr Val

$\begin{array}{lllll}1850 & 1860 & 1870 & 1880 & 1890\end{array}$

TTT ATC CGT GCC CAT GAT AAC AAC GTT CAA GAT ATT ATT GCT GAA ATC ATC Phe Ile Arg Ala His Asp Asn Asn Val Gln Asp Ile Ile Ala Glu Ile Ile

$$
\begin{array}{lllll}
1900 & 1910 & 1920 & 1930 & 1940
\end{array}
$$

AAG AAA GAA ATC AAT CCA AAA TCA GAT GGT TTC ACG ATT ACT GAT GCT GAA Lys Lys Glu Ile Asn Pro Lys Ser Asp Gly Phe Thr Ile Thr Asp Ala Glu

$$
\begin{array}{lllll}
1950 & 1960 & 1970 & 1980 & 1990
\end{array}
$$

ATG AAG CAA GCC TTT GAG ATT TAC AAC AAA GAC ATG CTC AGC AGC GAC AAA Met Lys Gln Ala Phe Glu Ile Tyr Asn Lys Asp Met Leu Ser ser Asp Lys

$$
\begin{array}{lllll}
2000 & 2010 & 2020 & 2030 & 2040
\end{array}
$$

AAA TAT ACG CTT AAC AAC ATC CCA GCG GCT TAC GCG GTT ATG TTG CAA AAC Lys Tyr Thr Leu Asn Asn Ile pro Ala Ala Tyr Ala Val Met Leu gln Asn

$$
20502060 \quad 2070 \quad 2080 \quad 2090
$$

ATG GAA ACT ATC ACT CGT GTC TAC TAT GGA GAC CTT TAT ACA GAT GAT GGT

Met Glu Thr Ile Thr Arg Val Tỳr Tyr Gly Asp Leu Tyr Thr Asp Asp Gly

$$
2100 \quad 2110 \quad 2120 \quad 2130 \quad 2140
$$

CAC TAC ATG GAA ACT AAG TCT CCA TAT TAC GAT ACC ATT GTT AAC TTG ATG His Tyr Met Glu Thr Lys Ser Pro Tyr Tyr Asp Thr Ile Val Asn Leu Met

$2150 \quad 2160 \quad 2170 \quad 2180 \quad 2190$

AAG AGT CGT ATC AAG TAT GTA TCT GGT GGG CAA GCA CAA CGT TCA TAC TGG Lys Ser Arg Ile Lys Tyr Val Ser Gly Gly Gln Ala Gln Arg Ser Tyr Trp

$220022210 \quad 220 \quad 2230 \quad 2240$

TTG CCA ACT GAT GGT AAG ATG GAC AAT TCA GAT GTT GAA CTT TAC CGC ACA

Leu Pro Thr Asp Gly Lys Met Asp Asn Ser Asp Val Glu Leu Tyr Arg Thr

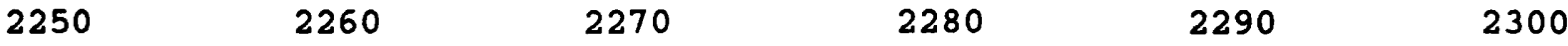

AAT GAA GTC TAC ACT TCA GTA CGT TAT GGT AAA GAC ATT ATG ACA GCT AAT

Asn Glu Val Tyr Thr Ser Val Arg Tyr Gly Lys Asp Ile Met Thr Ala Asn 
2310

2320

2330

2340

2350

GAT ACA GAA GGT TCT AAA TAC AGC CGT ACT TCT GGT CAG GTA ACA CTT GTA Asp Thr Glu Gly Ser Lys Tyr Ser Arg Thr ser Gly Gin Val Thr Leu Val

$\begin{array}{rrrrr}2360 & 2370 & 2380 & 2390 & 2400\end{array}$

GCT AAC AAT CCA AAA TTG AAT TTG GAT CAA TCA GCT AAA CTT AAT GTT GAA Ala Asn Asn Pro Lys Leu Asn Leu Asp Gln Ser Ala Lys Leu Asn Val Glu

$$
2410 \quad 2420 \quad 2430 \quad 2440 \quad 2450
$$

ATG GGT AAA ATC CAT GCC AAC CAA AAA TAC CGT GCT TTG ATT GTT GGT ACA Met Gly Lys Ile His Ala Asn Gln Lys Tyr Arg Ala Leu Ile Val Gly Thr

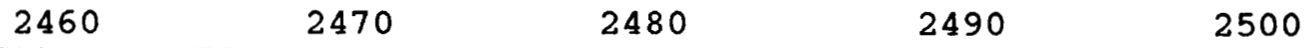

GCT GAT GGT ATC AAG AAC TTT ACA TCT GAT GCA GAT GCA ATC GCA GCA GGT Ala Asp Gly Ile Lys Asn Phe Thr Ser Asp Ala Asp Ala Ile Ala Ala Gly

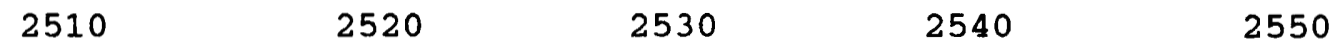

TAC GTT AAA GAA ACA GAC AGC AAC GGT GTC TTG ACT TTC GGT GCT AAT GAC Tyr Val Lys Glu Thr Asp Ser Asn Gly Val Leu Thr Phe Gly Ala Asn Asp

$25602570 \quad 2580 \quad 2590 \quad 2600$

ATC AAG GGT TAT GAA ACA TTT GAT ATG TCT GGT TTC GTA GCA GTT TGG GTT Ile Lys Gly Tyr Glu Thr Phe Asp Met ser Gly phe Val Ala Val Trp Val

$2610 \quad 2620 \quad 2630 \quad 2640 \quad 2650$

CCA GTT GGA GCT TCA GAT AAT CAA GAT ATC CGA GTA GCG CCT TCA ACA GAA Pro Val Gly Ala Ser Asp Asn Gln Asp Ile Arg Val Ala Pro Ser Thr Glu

$2660 \quad 2670 \quad 2680 \quad 2690 \quad 2700$

GCT AAA AAA GAG GGT GAA TTG ACT CTT AAA GCG ACT GAA GCT TAT GAT TCA Ala Lys Lys Glu Gly Glu Leu Thr Leu Lys Ala Thr Glu Ala Tyr Asp Ser

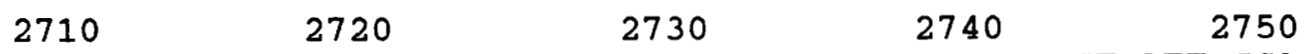

CAA TTA ATC TAC GAA GGC TTC TCT AAC TTT CAA ACT ATT CCA GAT GGT TCA Gln Leu Ile Tyr Glu Gly Phe Ser Asn Phe Gln Thr Ile Pro Asp Gly Ser

$\begin{array}{llllll}2760 & 2770 & 2780 & 2790 & 2800 & 2810\end{array}$
GAT CCT TCA GTC TAT ACT AAC CGT AAG ATT GCT GAA AAT GTT GAT TTG TTC Asp Pro Ser Val Tyr Thr Asn Arg Lys Ile Ala Glu Asn Val Asp Leu Phe

$$
2820 \quad 2830 \quad 2840 \quad 2850 \quad 2860
$$

AAA TCA TGG GGT GTA ACA TCA TTT GAA ATG GCA CCT CAA TTT GTA TCT GCT Lys Ser Trp Gly Val Thr Ser phe Glu Met Ala Pro Gln Phe val ser Ala

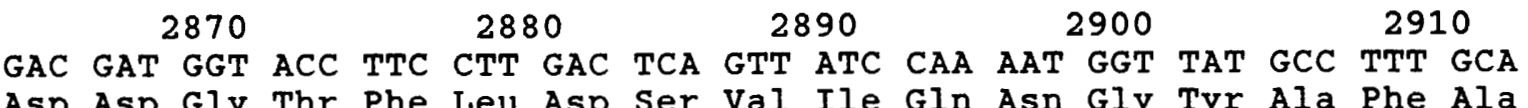

Asp Asp Gly Thr Phe Leu Asp Ser Val Ile Gln Asn Gly Tyr Ala Phe Ala

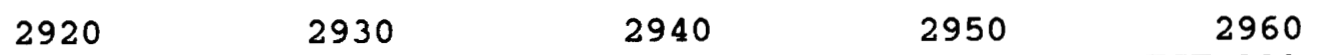

GAC CGT TAC GAT CTT GCC ATG AGT AAG AAC AAT AAA TAC GGT TCT AAA GAA Asp Arg Tyr Asp Leu Ala Met Ser Lys Asn Asn lys Tyr Gly ser Lys glu

$$
2970 \quad 2980 \quad 2990 \quad 3000 \quad 3010
$$

GAT CTA CGT GAT GCT CTT AAA GCA CTT CAT AAG GCT GGT ATT CAA GCA ATC Asp Leu Arg Asp Ala Leu Lys Ala Leu His Lys Ala Gly Ile Gln Ala Ile $\begin{array}{lllll}3020 & 3030 & 3040 & 3050 & 3060\end{array}$

GCT GAC TGG GTT CCA GAC CAA ATT TAC CAA TTG CCA GGT AAA GAA GTT GTA Ala Asp Trp Val pro Asp Gin Ile Tyr Gin Leu pro gly Lys Glu Val Val 
3070

3080

3090

3100

3110

ACA GCG ACT CGT ACT GAT GGT GCT GGT CGT AAG ATT GCG GAC GCT ATC ATT Thr Ala Thr Arg Thr Asp Gly Ala Gly Arg Lys Ile Ala Asp Ala Ile Ile

$\begin{array}{llllr}3120 & 3130 & 3140 & 3150 & 3160\end{array}$

GAC CAC TCA CTT TAT GTG GCT AAC TCT AAG TCA TCA GGC AAA GAT TAC CAA Asp His Ser Leu Tyr Val Ala Asn Ser Lys Ser Ser Gly Lys Asp Tyr Gln

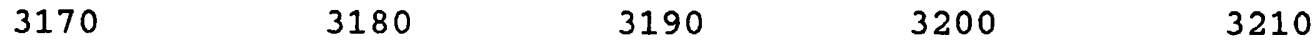

GCT AAA TAC GGT GGT GAA TTC TTG GCT GAA CTT AAA GCT AAG TAC CCT GAA Ala Lys Tyr Gly Gly Glu phe Leu Ala Glu Leu Lys Ala Lys Tyr pro Glu

$3220 \quad 3230 \quad 3240 \quad 3250 \quad 3260$

ATG TTC AAG GTA AAC ATG ATT TCA ACT GGT AAA CCA ATT GAT GAT TCT GTT

Met Phe Lys Val Asn Met Ile Ser Thr Gly Lys Pro Ile Asp Asp Ser Val

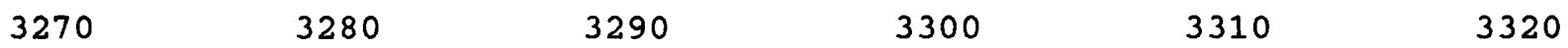

AAA TTG AAA CAA TGG AAG GCT GAA TAC TTC AAC GGA ACA AAC GTT CTT GAA Lys Leu Lys Gln Trp Lys Ala Glu Tyr Phe Asn Gly Thr Asn Val Leu Glu $\begin{array}{cccccc} & 3330 & 3340 & 3350 & 3360 & 3370 \\ \text { CGT GGT GTT GGC } & \text { TAT } & \text { GTA CTT } & \text { AGC GAT GAA } & \text { GCA } & \text { ACT } \\ \text { GGT AAG TAT } & \text { TTC ACT }\end{array}$ Arg Gly Val Gly Tyr Val Leu Ser Asp Glu Ala Thr Gly Lys Tyr Phe Thr

$3380 \quad 3390 \quad 3400 \quad 3410 \quad 3420$

GTC ACT AAA GAA GGT AAC TTC ATT CCT CTT CAA TTG ACA GGT AAA GAA AAG Val Thr Lys Glu Gly Asn phe Ile Pro Leu Gln Leu Thr Gly Lys Glu Lys

$\begin{array}{lllll}3430 & 3440 & 3450 & 3460 & 3470\end{array}$

GTT ATT ACT GGA TTC TCA AGT GAT GGT AAA GGA ATC ACT TAC TTC GGT ACA Val Ile Thr Gly Phe Ser Ser Asp Gly Lys Gly Ile Thr Tyr Phe Gly Thr

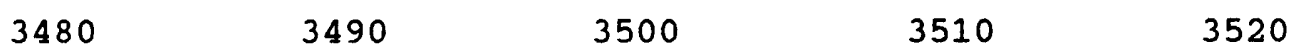

AGT GGT ACA CAA GCT AAA TCT GCC TTT GTA ACC TTC AAT GGT AAC ACT TAC Ser Gly Thr Gin Ala Lys Ser Ala Phe Val Thr phe Asn Gly Asn Thr Tyr

$$
\begin{array}{lllll}
3530 & 3540 & 3550 & 3560 & 3570
\end{array}
$$

TAC TTT GAT GCT CGT GGT CAC ATG GTT ACT AAC AGT GAA TAC TCA CCA AAT Tyr Phe Asp Ala Arg Gly His Met Val Thr Asn Ser Glu Tyr Ser Pro Asn

$\begin{array}{lllll}3580 & 3590 & 3600 & 3610 & 3620\end{array}$

GGT AAA GAC GTT TAT CGT TTC TTA CCA AAT GGT ATC ATG TTG AGT AAT GCC Gly Lys Asp Val Tyr Arg phe Leu Pro Asn Gly Ile Met Leu Ser Asn Ala

$\begin{array}{lllll}3630 & 3640 & 3650 & 3660 & 3670\end{array}$

TTC TAC ATT GAT GCT AAT GGT AAT ACC TAC CTT TAT AAC TCT AAA GGT CAA Phe Tyr Ile Asp Ala Asn Gly Asn Thr Tyr Leu Tyr Asn Ser Lys Gly Gln

$\begin{array}{lllll}3680 & 3690 & 3700 & 3710 & 3720\end{array}$

ATG TAC AAG GGT GGT TAC ACT AAA TTT GAT GTT TCT GAA ACT GAT AAA GAC Met Tyr Lys Gly Gly Tyr Thr Lys Phe Asp Val ser Glu Thr Asp Lys Asp

$\begin{array}{lllll}3730 & 3740 & 3750 & 3760 & 3770\end{array}$

GGT AAA GAA TCT AAG GTT GTG AAA TTC CGT TAC TTC ACT AAT GAA GGT GTC

Gly Lys Glu Ser Lys Val Val Lys Phe Arg Tyr Phe Thr Asn Glu gly Val

3780

$3790 \quad 3800 \quad 3810$

3820

3830

ATG GCC AAA GGT GTT ACG GTT ATT GAT GGT TTC ACA CAA TAT TTT GGA GAA

Met Ala Lys Gly Val Thr Val Ile Asp Gly Phe Thr Gln Tyr Phe Gly Glu 
3840

3850

3860

3870

3880

GAC GGT TTC CAA GCT AAA GAT AAG TTA GTA ACC TTT AAA GGT AAA ACT TAT Asp Gly Phe Gln Ala Lys Asp Lys Leu Val Thr Phe Lys Gly Lys Thr Tyr

$\begin{array}{lllll}3890 & 3900 & 3910 & 3920 & 3930\end{array}$

TAC TTT GAC GCA CAC ACT GGT AAT GGT ATC AAG GAT ACT TGG AGA AAT ATC Tyr Phe Asp Ala His Thr Gly Asn Gly Ile Lys Asp Thr Trp Arg Asn Ile

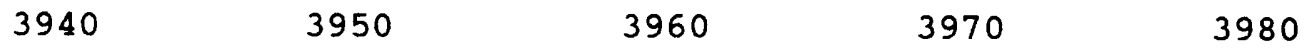

AAT GGT AAG TGG TAC TAC TTT GAT GCA AAC GGT GTT GCT GCT ACA GGT GCA Asn Gly Lys Trp Tyr Tyr phe Asp Ala Asn Gly Val Ala Ala Thr Gly Ala

3990400004020030

CAA GTC ATC AAT GGT CAA AAA CTT TAC TTC AAT GAA GAT GGA AGC CAA GTT Gln Val Ile Asn Gly Gln Lys Leu Tyr Phe Asn Glu Asp Gly ser Gln Val

$4040 \quad 4050 \quad 4060 \quad 4070 \quad 4080$

AAA GGT GGC GTT GTT AAG AAT GCA GAT GGT ACT TAC AGC AAG TAC AAA GAA Lys Gly Gly Val Val Lys Asn Ala Asp Gly Thr Tyr Ser Lys Tyr Lys Glu

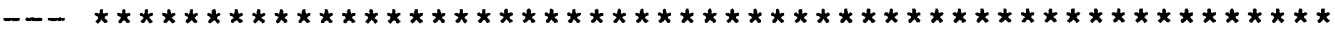

$4090 \quad 4100 \quad 4110 \quad 4120 \quad 4130$

GGT TTT GGA GAG CTA GTG ACT AAC GAA TTC TTC ACA ACT GAT GGC AAT GTT Gly Phe Gly Glu Leu Val Thr Asn Glu Phe Phe Thr Thr Asp Gly Asn Val

$\begin{array}{lllll}4140 & 4150 & 4160 & 4170 & 4180\end{array}$

TGG TAC TAT GCA GGC GCT AAT GGT AAG ACT GTT ACA GGT GCA CAA GTC ATC Trp Tyr Tyr Ala Gly Ala Asn Gly Lys Thr Val Thr Gly Ala Gln Val Ile

$\begin{array}{lllll}4190 & \mathbf{4 2 0 0} & \mathbf{4 2 1 0} & \mathbf{4 2 2 0} & \mathbf{4 2 3 0}\end{array}$

AAT GGC CAA CAC CTA TAC TTT AAT GCA GAC GGA AGC CAA GTT AAG GGT GGT Asn Gly Gln His Leu Tyr Phe Asn Ala Asp Gly Ser Gln Val Lys Gly Gly

$\begin{array}{lllll}4240 & 4250 & 4260 & 4270 & 4280\end{array}$

GTT GTT AAG AAT GCA GAT GGT ACT TAT AGT AAG TAT AAT GCT TCA ACA GGT Val Val Lys Asn Ala Asp Gly Thr Tyr Ser Lys Tyr Asn Ala ser Thr Gly

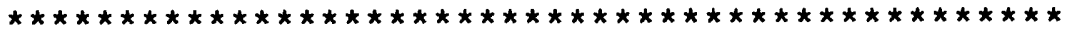

$\begin{array}{llllll}\mathbf{4 2 9 0} & \mathbf{4 3 0 0} & \mathbf{4 3 1 0} & \mathbf{4 3 2 0} & \mathbf{4 3 3 0} & \mathbf{4 3 4 0}\end{array}$
GAA CGC TTG ACT AAT GAG TTT TTC ACA ACA GGC GAC AAC AAC TGG TAC TAC Glu Arg Leu Thr Asn Glu phe Phe Thr Thr Gly Asp Asn Asn Trp Tyr Tyr

$$
4350 \quad 4360 \quad 4370 \quad 4380 \quad 4390
$$

ATT GGT GCT AAT GGT AAG TCA GTG ACT GGT GAA GTT AAA ATT GGT GAC GAT Ile Gly Ala Asn Gly Lys Ser Val Thr Gly Glu Val Lys Ile Gly Asp Asp

$4400 \quad 4410 \quad 4420 \quad 04300$

ACT TAT TTC TTC GCT AAG GAT GGT AAA CAA GTA AAA GGT CAA ACA GTA AGT Thr Tyr Phe Phe Ala Lys Asp Gly Lys Gln Val Lys Gly Gln Thr Val Ser

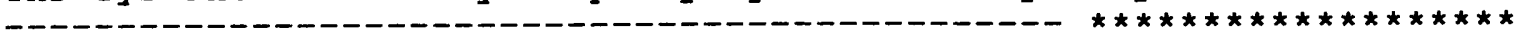

$4450 \quad 4460 \quad 4470 \quad 4480 \quad 44 \pi x$

GCT GGC AAT GGT CGA ATT AGC TAT TAC TAT GGT GAT AGT GGT AAG AGA GCT Ala Gly Asn Gly Arg Ile Ser Tyr Tyr Tyr Gly Asp Ser Gly Lys Arg Ala

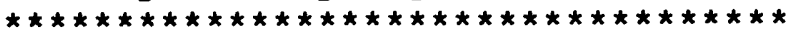

$$
\begin{array}{lllll}
4500 & 4510 & 4520 & 4530 & 4540
\end{array}
$$

GTT AGC ACA TGG ATA GAA ATT CAA CCA GGA GTT TAC GTT TAC TTT GAT AAG Val Ser Thr Trp Ile Glu Ile Gln Pro Gly Val Tyr Val Tyr phe Asp Lys

$\begin{array}{llllll}4550 & 4560 & 4570 & \mathbf{4 5 8 0} & \mathbf{4 5 9 0} & \mathbf{4 6 0 0}\end{array}$ AAT GGT CTT GCT TAT CCA CCT AGA GTG CTA AAC TAA TTGATATAAA Aaggaggact Asn Gly Leu Ala Tyr Pro Pro Arg Val Leu Asn End 


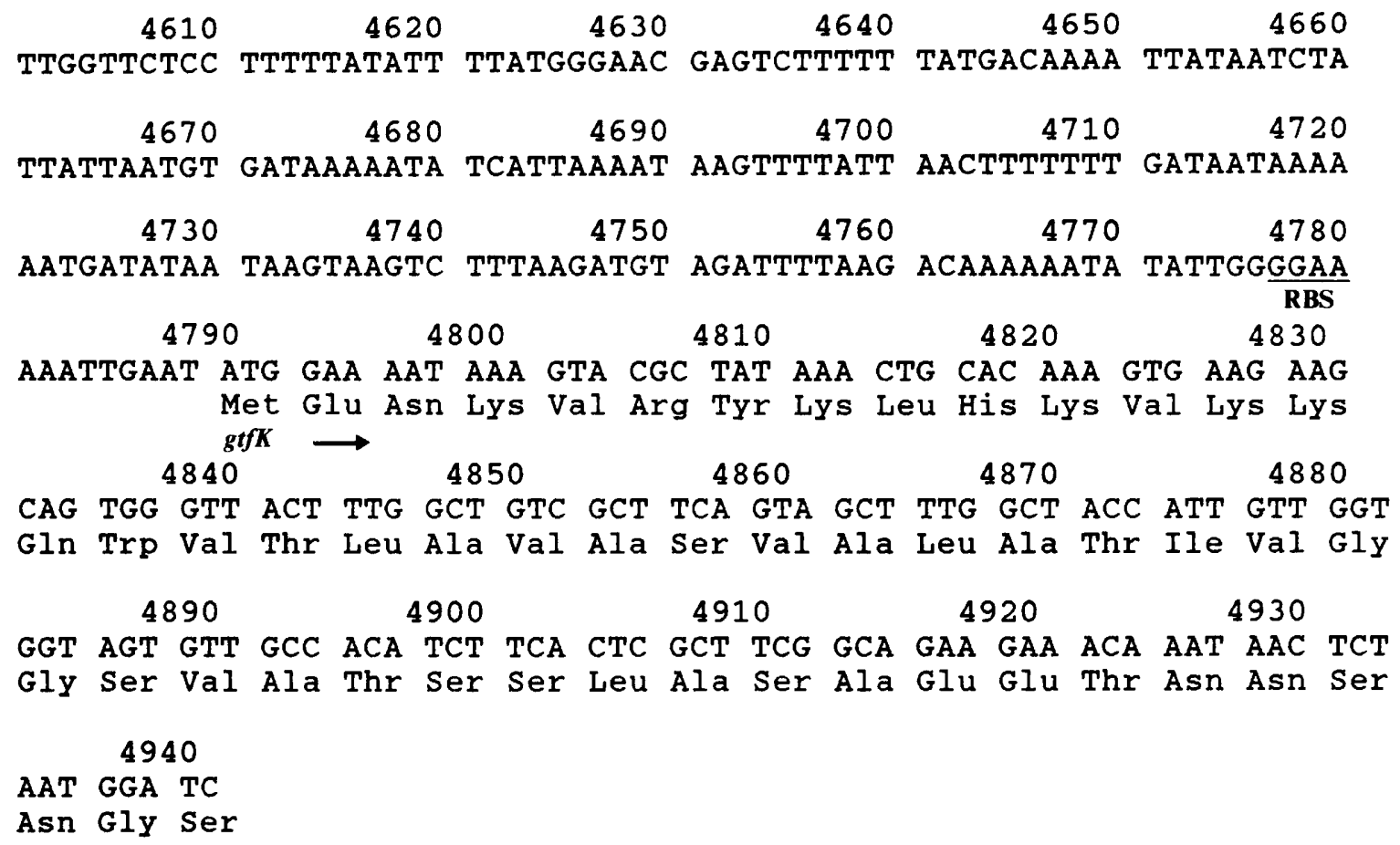

\section{Results}

\section{Analysis of restriction maps of $\lambda A-8$ and $\lambda A-33$}

Restriction maps of $\lambda A-8$ coding for a GTF-I activity and $\lambda A-33$ coding for a GTF-S activity were constructed using double restriction digests, and confirmed by sequential deletion of restriction fragments using nuclease Bal31 (Fig. $1 a, b$ ).

Examination of the restriction maps of the two $\lambda$-clones revealed an apparent overlap in which the two cloned fragments of DNA could be depicted as a contiguous locus (Fig. 1c). The overlap was confirmed by probing $E c o$ RI digests of $\lambda \mathrm{A}-8$ and $\lambda \mathrm{A}-33$ under highly stringent conditions with the purified $1.9 \mathrm{kbp}$ or the $0.9 \mathrm{kbp} E c o$ RI fragments from the right-hand end of $\lambda$ A-8 clone (i.e. the EcoRI fragments from within the overlapping region; Fig. 1). Identical signals were obtained from the $\lambda \mathrm{A}-8$ and $\lambda \mathrm{A}-33 E c o$ RI digests (data not shown), indicating that the $0.9 \mathrm{kbp}$ and $1.9 \mathrm{kbp}$ $E c o$ RI fragments in $\lambda \mathrm{A}-8$ and $\lambda \mathrm{A}-33$ were homologous, and that the clones are indeed overlapping.

\section{Hybridizations with the $S$. salivarius chromosome}

To ensure that no cloning artifacts had occurred when $\lambda A-8$ and $\lambda A-33$ were constructed, Southern hybridization into the $S$. salivarius chromosome was carried out. $\lambda \mathrm{A}-8, \lambda \mathrm{A}-33$ and $S$. salivarius genomic DNA cleaved with $S a c I$ and $B g l$ II were probed after electrophoresis with the $1.9 \mathrm{kbp} E c o$ RI fragment from $\lambda \mathrm{A}-8$. A SacI and $B g l \mathrm{II}$ double digest was chosen because it was expected to yield two easily resolvable fragments that encompassed nearly the entire locus. The probe was chosen because it spanned the junction between the two fragments (Fig. $2 a$ ). As predicted, the two hybridizing bands in the $S$. salivarius DNA lane were of the same $M_{\mathrm{r}}$ as the single hybridizing bands in the $\lambda \mathrm{A}-8$ and $\lambda \mathrm{A}-33$ lanes (Fig. $2 b$ ). This indicated that the contiguous locus depicted in Fig. $1(c)$ exists in the $S$. salivarius genome.

\section{Subcloning of the gtf genes}

Prior SDS-PAGE experiments indicated that both gene products have $M_{\mathrm{r}}$ values of 160000 or greater (unpublished observations). It can be inferred from this that the coding regions are greater than $4.5 \mathrm{kbp}$ so appropriately large fragments were subcloned into the phagemid vector pIBI30. Those $E$. coli strains harbouring plasmids carrying either the $6.5 \mathrm{kbp} \mathrm{SacI-BamHI}$ fragment from $\lambda A-8$ (pGS101) or the $6.0 \mathrm{kbp} \mathrm{BglII-BamHI}$ fragment from $\lambda A-33$ (pGS201) (Fig. 1d) were positive for GTF activity. SDS-PAGE analysis could not distinguish between the products of the subclones and those of the parent $\lambda$-clones (Fig. 3). 


\section{Sequence analysis of the gtfJ gene}

The nucleotide sequence of the region from $44 \mathrm{bp}$ to the left of the leftmost HindIII site to the end of the insert in pGS101 some 4941 bp to the right was determined (Fig. 1e). An open-reading frame of $4566 \mathrm{bp}$ beginning with a codon for methionine was located 9 bp downstream from a putative ribosome-binding site (Fig. 4). The deduced amino acid sequence of this open-reading frame defined a highly hydrophilic protein with an $M_{\mathrm{r}}$ of 167717 , consistent with previous biochemical studies of the GTF-I of $S$. salivarius (see above). The amino-terminus consisted of a 19-amino-acid basic domain followed by a 21-amino-acid hydrophobic domain characteristic of a signal sequence required for export (von Heijne, 1990).

\section{Sequence comparisons with gtf genes from other oral streptococci}

All $g t f$ genes from oral streptococci thus far sequenced have proved to be very similar. The deduced amino acid sequence of the gtfB gene from $S$. mutans GS5, which encodes a GTF-I enzyme, was chosen as a 'typical' $g t f$ gene for comparison with the deduced amino acid sequence of the $g t f J$ gene of $S$. salivarius. The sequences were aligned using the FastP program (Pearson, 1990) and displayed $40 \%$ identity overall (Fig. 5). The regions of greatest similarity were between GtfJ residues 420 and 480,920 and 1020 , and 1040 and 1110 , and this is consistent with previously reported sequence comparisons of GTFs from mutans streptococci (Ferretti et al., 1990). Interestingly, a major discontinuity was observed in aligning the $\mathrm{GtfB}$ sequence since 47 amino acids in the GtfJ sequence had no counterpart in the GtfB protein (Fig. 5). No simple explanation for this observation was forthcoming.

The carboxy-terminal domains of all streptococcal GTFs thus far sequenced possess a series of direct repeats that are thought to be involved in glucan binding (Ferretti et al., 1987; Wong et al., 1990). The bestcharacterized repeated sequence is the co-called 'AC' repeat for which a 54-amino-acid consensus sequence has been deduced (Gilmore et al., 1990). In this 'AC' repeat the first 33 amino acids constitute the ' $A$ ' region and the final 21 amino acids the ' $C$ ' region. We have found substantial similarity with the ' $A$ ' region coded by three locations in $g t f J$ [beginning with nucleotides 3942 (A1), 4137 (A2) and 4332 (A3) in Fig. 4]. The deduced amino acid sequences of these regions in $g t f J$ show $58 \%$, $52 \%$ and $40 \%$ identity respectively to the amino acid consensus sequence. Interestingly, the 14 amino acid residues following $\mathrm{A} 1$ and $\mathrm{A} 2$ in GtfJ were $100 \%$ identical to each other but bore no resemblance to the ' $\mathrm{C}$ ' region of the consensus sequence. We have termed these 14 residues the ' $D$ ' repeat. There was also a weakly conserved ' $D$ ' repeat immediately following A3 (Fig. 4).

\section{Location of the gtfK gene}

The hybridization and subcloning evidence outlined above indicates that the $g t f K$ gene, which encodes a GTF-S activity, lies close to the $g t f J$ gene. We observed that the extreme 3 '-OH end of the nucleotide sequence in Fig. 4 was similar to the beginning of the $g t f J$ gene. This similarity started at a methionine codon (nucleotides 4790-4792 in Figs 4 and 6) and was preceded by a possible ribosome-binding site (Fig. 4). We therefore hypothesized that this was the beginning of the gtfK gene. To provide supporting evidence for this, the nucleotide sequence of a $150 \mathrm{bp}$ region approximately $2.7 \mathrm{kbp}$ downstream was determined. This region was chosen for two reasons. First, all sequenced streptococcal $g t f$ genes are conserved in this area (cf. Fig. 5; Gilmore $e t$ al., 1990). Secondly this area should represent a region approximately at the centre of the $g t f K$ coding region as the size of all Gtfs predetermines the need for an openreading frame in excess of $4.5 \mathrm{kbp}$ (see above). The sequence approximately $2.7 \mathrm{kbp}$ downstream was found to be $81 \%$ identical to the region beginning at $g t f J$ nucleotide 2775 (Fig. 6). This evidence strongly supports the notion that the gtf $K$ gene commences $211 \mathrm{bp}$ downstream from the translation stop codon of the $g t f J$ gene and that both genes are transcribed in the same direction (Fig. 1f).

\section{Discussion}

This study has shown that the gtf $J$ gene which encodes a GTF-I activity and the $g t f K$ gene which encodes a GTF-S activity are located very close to each other on the S. salivarius ATCC 25975 chromosome. The gtfJ gene is upstream of the $g t f K$ gene and both genes appear to be transcribed in the same direction. The proximity and gene order of $g t f J$ and $g t f K$ are reminiscent of those found with the $g t f B$ and $g t f C$ genes of $S$. mutans GS5, which encode a GTF-I and GTF-S respectively. However, while in $S$. salivarius the products of both the $g t f J$ and $g t f K$ genes are primer-dependent (Pitty et al., 1989), that of the $g t f C$ gene is primer-independent (Ueda et al.,

Fig. 5. Alignment of the $S$. salivarius GtfJ with the GtfB of $S$. mutans. The alignment was generated using the FastP program. Two separate alignments were generated, one from each side of the discontinuity at GtfB residue 679 and the two alignments were merged to form the final figure. 
$10 \quad 20 \quad 30 \quad 40 \quad 50$

GHJ MENKIHYKLHKVKKQWVTIAVASVALATVLGGLSVTTSSVSADETQDKTVTQSNS

GtB $\quad \begin{aligned} & \text { MDKKVRYKLRKVKKRWVTVSVASAVM--TLTTLS--GGLVKADSNESK-SQISN } \\ & \text { M }\end{aligned}$ $\begin{array}{llll}10 & 20 & 30 & 40\end{array}$

$\begin{array}{llllll}60 & 70 & 80 & 90 & 100 & 110\end{array}$

GTTASLVTSPEATKEADKRTNTKEADVLTPAKETNAVETATTTNTQATAEAATTATTADV DSNTSVVTANE---ESNVITEATSKQEAASSQTNHTV---TTSSSSTSVVNPKEVVSNPY $\begin{array}{cccccc}50 & 60 & 70 & 80 & 90 & 100\end{array}$
120
130
140
150
160
170

AVAAVPNKEAVVTTDAPAVTTEKAEEQPATVKAEVVNTEVKAPEAALKDSEVEAALSLKN $. . \ldots \ldots \ldots . \ldots \ldots . \ldots . \ldots \ldots \ldots \ldots \ldots \ldots . \ldots \ldots . .$. TVGETASNGEKLQNQTTTV-DKT SEAAANNISKQTTEADTDVIDDS-NAANLQILEKLPN 110 120

130

140

150 160

180 190

200

210

220

230

IKNIDGKYYYVNEDGSHKENFAITVNGQLLYFGKDGALTSSSTYSFTPG-TTNIVDGFSI

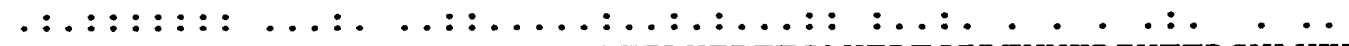
VREIDGKYYYYDNNGKVRTNFTLIADGKILHFDETGAYTDTS IDTVNKDIVTTRSNLYKK 170 180 190 200 210 220
240
250
260
270
280
290

NNRAYDSSEASFELIDGYLTADSWYRPASIIKDGVTWQASTAEDFRPLLMAWWPNVDTQV

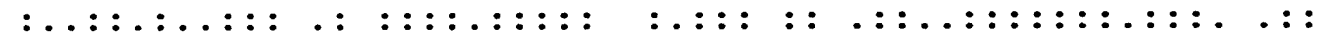
YNQVYDRSAQSFEHVDHYLTAESWYRPKYILKDGKTWTQSTEKDFR PLLMTWWPDQETQR 230 240 250 260 270 280

$$
300
$$
310 320 330 340 350

NYLNYMSKVFNLDAKYSSTDKQETLKVAAKDIQIKIEQKIQAEKSTQWLRETISAFVKTQ

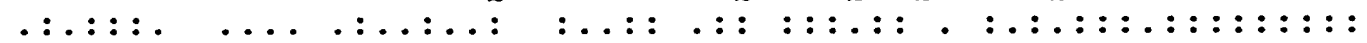
QYVNYMNAQLGINKTYDDTSNQLQLNIAAATIQAKIEAKITTLKNTDWLRQTISAFVKTQ 290 300 310 320 330 340

360 370 380 390 400 410 PQWNKETENYSKGGGEDHLQGGALLYVNDSR-TPWANSDYRRLNRTATNQTGTIDKSILD ..: : . . :

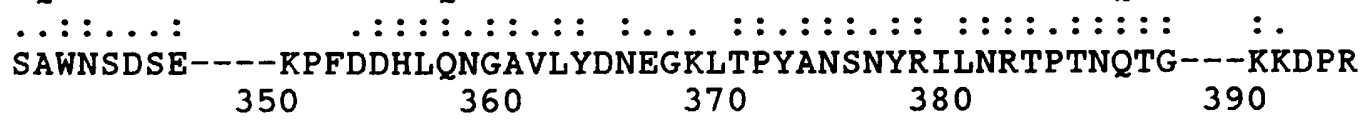
420 430 440 450 460 470 EQSDPNHMGGFDFLLANDVDLSNPVVQAEQLNQIHYLMNWG SIVMGDKDANFDGIRVDAV

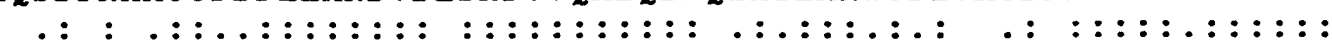
YTAD-NTIGGYEFLLANDVDNSNPVVQAEQLNWLHFLMNF GNIYANDPDANFDSIRVDAV 400 410 420 430 440 450

480 490 500 510

520 530 DNVDADMLQLYTNYFREYYGVNRSEANALAHI SVLEAWSLNDNHYNDKTDGAALAMENKQ

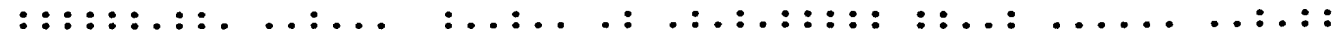
DNVDADLLQIAGDYLKAAKG IHKNDKAANDHLS ILEAWSDNDTPYLHDDGDNMINMDNKL 460 470 480 490 500 510 
540

550

560

570

580

590

RLALLFSLAKPIKERTPAVSPLYNNTFNTTQRDEKTDWINKDGSKAYNEDGTVKQSTIGK

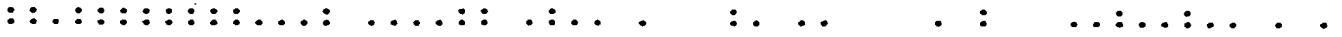
RLSLLF SLAKPLNQR-SGMNPLITNSLVNRTDDNAETAAVP SYSF IRAHDSEVQDL IADI 520 530 540 550 560 570

600 610 620 630 640 YNEKYGDASGNYVF IRAHDNNVQDI----IAEI IKKEINPKSD-GFTITDAEMKQAFEIY

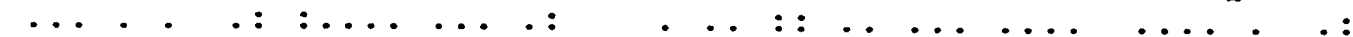
IKAEINPNVVGYSFTMEEIKKAFEIYNKDLLATEKKYTHYNTALSYALLLTNKS SVPRVY 580 590 600 610 620 630

650 670

680 690 700

NKDMLS SDKKYTLNNIPAAYAVMLQNMETITRVYYGDLYTDDGHYMETKS PYYDTIVNLM $:$ : : : : YGDMFTDDGOY-MAHKTINYEAIETLLKAR IKYVSGGOAMRNOQ $\begin{array}{llll}640 & 650 & 660 & 670\end{array}$

710 720 730 740 750 760

KSR IKYVSGGQAQR SYWLPTDGKMDNSDVELYRTNEVYT SVRYGKDIMTANDTEGSKYSR

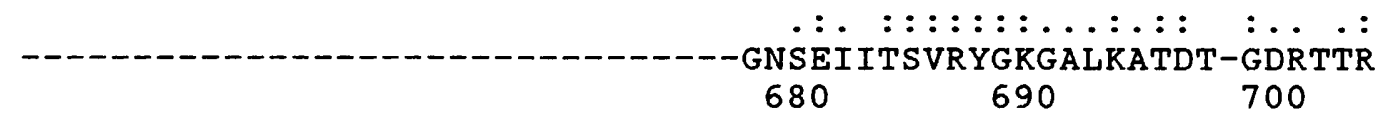
$\begin{array}{llllll}770 & 780 & 790 & 800 & 810 & 820\end{array}$

TSGQVTLVANNPKLNLDQSAKLNVEMGKIHANQKYRALIVGTADG IKNFT SDADAIAAGY

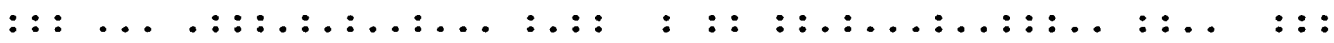
TSGVAVIEGNNPSLRLKASDRVVVNMGAAHKNQAYRPLLLTTDNGIKAYHSDQE--AAGL 710 720 730

740 750 760

\section{$\begin{array}{llllll}830 & 840 & 850 & 860 & 870 & 880\end{array}$}

VKETDSNGVLTFGANDIKGYETFDMSGFVAVWVPVGASDNQDIRVAP STEAKKEGEL-TL

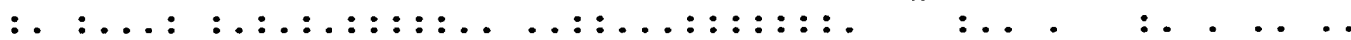
VRYTNDRGELIFTAADIKGYANPQVSGYLGVWVPVGAA---LIKMFALRLARPHQQMASV 770 780 790 800 810
890
900
910
920
930
940

KATEAYDSQLIYEGF SNFQTIPDGSDPSVYTNRKIAENVDLFRSWGVTSFEMAPQFVSAD

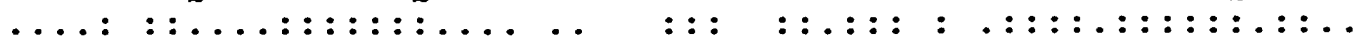
HQNAALDSRVMFEGF SNFQAFATKRE--EYTNVVIAKNVDKFAEWGVTDFEMAPQYVSST 820 830 840 850 860 870

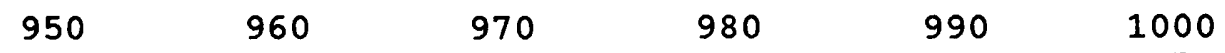
DGTFLDSVIQNGYAFADRYDLAMSKNNKYG SKEDLRDALKALHKAGIQAIADWVPDQIYQ

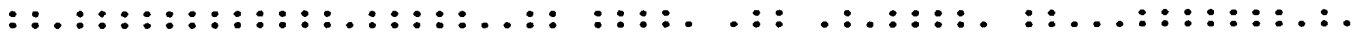
DGSFLDSVIQNGYAFTDRYDLGISKPNKYGTADDLVKAIKALHSKG IKVMADWVPDQMYA $\begin{array}{llllll}880 & 890 & 900 & 910 & 920 & 930\end{array}$

\section{$\begin{array}{llllll}1010 & 1020 & 1030 & 1040 & 1050 & 1060\end{array}$}

LPGKEVVTATRTDGAGRKIADAIIDHSLYVANSKS SGKDYQAKYGGEFLAELKAKYPEMF

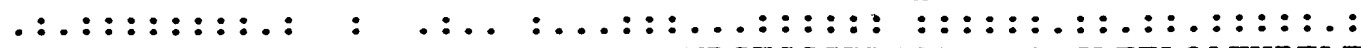
FPEKEVVTATRVDKYGTPVAGSQIKNTLYVVDGKSSGKDQQAKYGGAFLEELQAKYPELF $\begin{array}{llllll}940 & 950 & 960 & 970 & 980 & 990\end{array}$ 


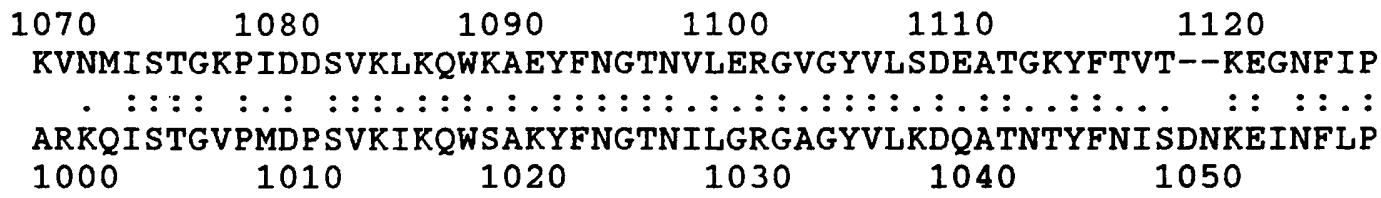

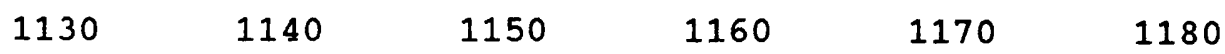

LQLTGKEKVITGF S SDGKGITYFGTSGTQAKSAFVTFNGNTYYFDARGHMVTNSEYSPNG

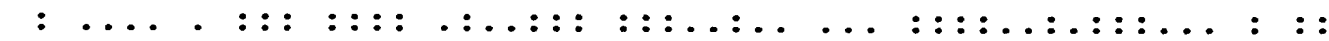
KTLLNQDSQV-GFSYDGKGYVYYST SGYQAKNTF ISEGDKWYYFDNNGYMVTGAQ-S ING $\begin{array}{llllll}1060 & 1070 & 1080 & 1090 & 1100 & 1110\end{array}$

$\begin{array}{llllll}1190 & 1200 & 1210 & 1220 & 1230 & 1240\end{array}$

KDVYRFLPNGIMLSNAFYIDANGNTYLYNSKGQMYKGGYTKFDVSETDKDGKESKVVKFR

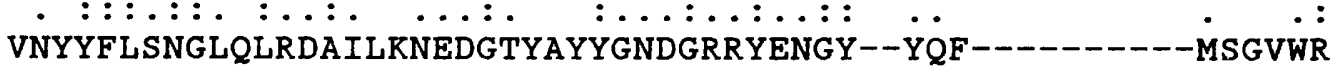
$\begin{array}{lllll}1120 & 1130 & 1140 & 1150 & 1160\end{array}$

$\begin{array}{llllll}1250 & 1260 & 1270 & 1280 & 1290 & 1300\end{array}$ YFTNEGVMAKGVTVIDGFTQYFGEDGFQAKDKLVTF-KGKTYYFDAHTGNGIKDTW-RNI

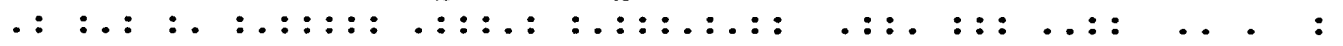
HF-NNGEMSVGLTVIDGQVQYFDEMGYQAKGKFVTTADGKIRYFDKQSGNMYRNRF IENE 1170 1180 1190 1200 1210 1220

1310 1320

1330

1340

1350

1360

NGKWYYFDANGVAATGAQVINGQKLYFNEDGSQVKGGVVKNADGTY SKYKEGFGELVTNE

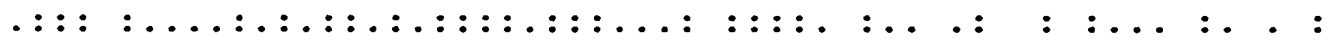
EGKWLYLGEDGAAVTGSQTINGQHLYFRANGVQVKGEFVTDHHGR ISYYDGNSGDQIRNR
1230
1240
1250
1260
1270
1280
1370
1380
1390
1400
1410
1420

FFTTDGNVWYYAGANGKTVTGAQVINGQHLYFNADGSQVKGGVVKNADGTYSKYNASTGE

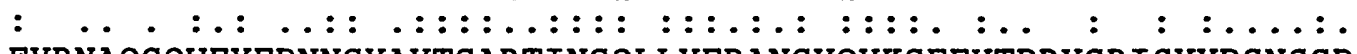
FVRNAQGQWFYFDNNGYAVTGARTINGQLLYFRANGVQVKGEFVTDRYGRISYYDGNSGD $\begin{array}{llllll}1290 & 1300 & 1310 & 1320 & 1330 & 1340\end{array}$
1430
1440
1450
1460
1470
1480

RLTNEFFTTGDNNWYYIGANGKSVTGEVKIGDDTYFFAKDGKQVKGQTVSAGNGRISYYY

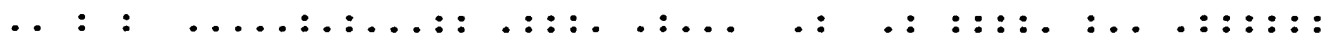
QIRNRFVRNAQGQWFYFDNNGYAVTGARTINGQHLYFRANGVQVKGEFVTDRHGRISYYD 1350 1360 1370 1380

1390 1400

$1490 \quad 1500 \quad 1510$

GDSGKRAVSTWIEIQPGVYVYFDKNGLAYP PRVLN

$:$ : :. . . . .: . : : : : : :

GNSGDQIRNRFVRNAQGQWFYFDNNGYAVTGARTINGQHLYFRANGVQVKGEFVTDRYGR $\begin{array}{llllll}1410 & 1420 & 1430 & 1440 & 1450 & 1460\end{array}$ 


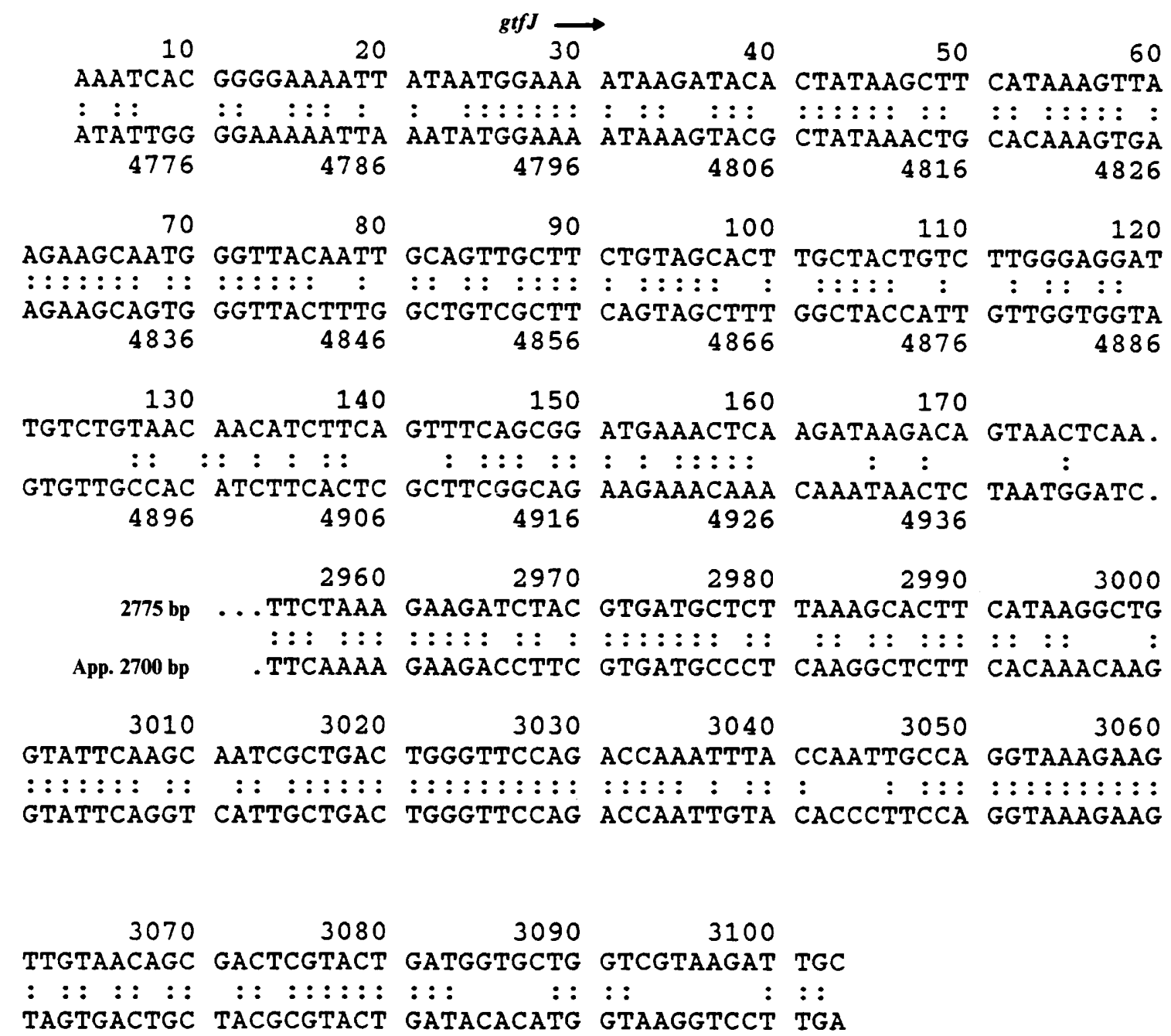

Fig. 6. Alignment of two regions of $g t f J$ with the putative homologous regions of $g t f K$. This alignment was generated using the IBI-Pustell DNA sequence analysis software. The downstream portion of $g t f K$ shown here has been submitted to GenBank and is listed under accession number M64112.

1988) and apparently produces insoluble as well as soluble products.

Pairwise sequence alignments (data not shown) have indicated that the $g t f J$ gene of $S$. salivarius is about as closely related to the sequenced $g t f$ genes from the mutans streptococci as the genes from the mutans group are to each other (Gilmore et al., 1990; Honda et al., 1990). Preliminary data also indicate that this is also likely to be the case for the $g t f K$ gene of $S$. salivarius. Since $S$. salivarius is not included in, or considered to be closely related to, the mutans group (Schleifer \& KilpperBälz, 1987; Coykendall, 1989) it can be inferred that either some sort of horizontal gene transfer has occurred in the oral environment, or that there is a strong constraint on the sequence of GTFs. These questions remain to be resolved.

One implication of a close similarity between the GTFs from mutans streptococci and the GTFs from
$S$. salivarius is that $S$. salivarius may have a more important role in caries aetiology than is generally assumed. This organism is present in very large numbers on the dorsal surface of the tongue and in the saliva (Gibbons \& van Houte, 1978). Nearly all strains synthesize extracellular GTFs (Eifuku et al., 1989) and there seems to be no reason why these enzymes should not form part of the acquired protein pellicle on the tooth surface and contribute significantly to caries aetiology (Rölla et al., 1983). Thus, even though the organisms themselves may not always be found in large numbers in plaque, the GTFs they produce may absorb to the tooth surface since GTF absorption and polymer formation are generally thought to precede bacterial colonization (Smith \& Taubman, 1978).

The regulation of expression of GTFs in S. salivarius ATCC 25975 is the subject of ongoing investigations in our laboratory (Markevics \& Jacques, 1985; Pitty \& 
Jacques, 1987). It has been found that the level of synthesis and secretion of both GTF-I and GTF-S activities can be increased by a perturbation of the membrane lipid environment. This has led to the hypothesis that regulation of expression occurs at the level of protein translocation across the cytoplasmic membrane. The proximity of the $g t f J$ and $g t f K$ genes in $S$. salivarius and the co-ordinate regulation of GTF-I and GTF-S activities observed in vitro (Pitty \& Jacques, 1987) suggest that these genes may form an operon. This is currently the subject of investigation.

This work was supported by a Program Grant awarded by the Australian National Health and Medical Research Council and in part by the Australian Dental Research Fund Inc.

\section{References}

Aduse-OpoKu, J., Gilpin, M. L. \& Russell, R. R. B. (1989). Genetic and antigenic comparison of Streptococcus mutans fructosyltransferase and glucan-binding protein. FEMS Microbiology Letters 50, 279-282.

Coykendall, A. L. (1989). Classification and identification of the viridans streptococci. Clinical Microbiology Reviews 2, 315-328.

Eifuku, H., Yoshimitsu-Narita, A., Sato, S., YakushiJi, T. \& Inoue, M. (1989). Production and partial characterization of the extracellular polysaccharides from oral Streptococcus salivarius. Carbohydrate Research 194, 247-260.

Dretzen, G., Bellard, M., Sassone-Corsi, P. \& Chambon, P. (1981). A reliable method for the recovery of DNA fragments from agarose and acrylamide gels. Analytical Biochemistry 112, 295-298.

Drucker, D. B., Shakespeare, A. P. \& Green, R. M. (1984). The production of dental plaque and caries by the bacterium Streptococcus salivarius in gnotobiotic WAG/RIJ rats. Archives of Oral Biology 29, 437-443.

Ferretti, J. J., Gilpin, M. L. \& Russell, R. R. B. (1987). Nucleotide sequence of a glucosyltransferase gene from Streptococcus sobrinus MFe28. Journal of Bacteriology 169, 4271-4278.

GibBons, R. J. \& VAN HoUTE, J. (1978). Oral bacterial ecology. In Textbook of Oral Biology, pp. 684-705. Edited by J. H. Shaw, E. A. Sweeney, C. C. Cappuccino \& S. M. Mellor. Philadelphia: W. B. Saunders.

GILMORE, K. S., RUSSELl, R. R. B. \& FerRetTI, J. J. (1990). Analysis of the Streptococcus downei gtfS gene, which specifies a glucosyltransferase that synthesizes soluble glucans. Infection and Immunity 58, 2452-2458.

Gough, J. A. \& Murray, N. E. (1983). Sequence diversity among related genes for recognition of specific targets in DNA molecules. Journal of Molecular Biology 166, 1-19.

Hamilton, I. R. (1967). Synthesis and degradation of intracellular polyglucose in Streptococcus salivarius. Canadian Journal of Microbiology 14, 65-77.

von Heijne, G. (1990). The signal peptide. Journal of Membrane Biology 115, 195-201.

HeNIKOFF, S. (1984). Unidirectional digestion with exonuclease III creates targeted breakpoints for DNA sequencing. Gene 28, 351-359.

Honda, O., Kato, C. \& Kuramitsu, H. K. (1990). Nucleotide sequence of the Streptococcus mutans gtfD gene encoding the glucosyltransferase-S enzyme. Journal of General Microbiology 136, 2099-2105.
Krasse, B. \& Carlsson, J. (1970). Various types of streptococci and experimental caries in hamsters. Archives of Oral Biology 15, 25-32.

LOENEN, W. A. \& BRAMmaR, W. J. (1980). A bacteriophage lambda vector for cloning large DNA fragments made with several restriction enzymes. Gene 10, 249-259.

Maniatis, T., Fritsch, E. F. \& SAmbrook, J. (1982). Molecular Cloning: A Laboratory Manual, Cold Spring Harbor, NY: Cold Spring Harbor Laboratory.

MARKEVICS, L. J. \& JACQUES, N. A. (1985). Enhanced secretion of glucosyltransferase by changes in potassium ion concentrations is accompanied by an altered pattern of membrane fatty acids in Streptococcus salivarius. Journal of Bacteriology 161, 989-994.

Murray, N. E., Brammar, W. J. \& MurRay, K. (1977). Lambdoid phages that simplify the recovery of in vitro recombinants. Molecular and General Genetics 150, 53-61.

PeARson, W. R. (1990). Rapid and sensitive sequence comparison with FastA and FastP. Methods in Enzymology 188, 63-98.

Perry, D. \& Kuramitsu, H. K. (1990). Linkage of sucrosemetabolizing genes in Streptococcus mutans. Infection and Immunity 58, 3462-3464.

Pitty (née Markevics), L. J. \& JacQues, N. A. (1987). The influence of incorporation of octadecenoic acid on the cell-associated fructosyltransferase and the extracellular glucosyltransferase activities of Streptococcus salivarius. Journal of General Microbiology 133, 3565-3573.

Pitty, L. J., Giffard, P. M., Gilpin, M. L., Russell, R. R. B. \& JACQUES, N. A. (1989). Cloning and expression of glycosyltransferase activities from Streptococcus salivarius. Journal of Dental Research 68 , 1681-1682.

Rölla, G., Ciardi, J. E., Eggen, K. H., Bowen, W. F. \& Afseth, J. (1983). Free glucosyl- and fructosyl-transferase in human saliva and adsorption of these enzymes to teeth in vivo. In Proceedings: Glucosyltransferase, Glucans, Sucrose and Dental Caries. Special Supplement to Chemical Senses, pp. 21-29. Edited by J. R. Doyle \& J. E. Ciardi. Oxford: IRL Press.

SANGER, F., Nicklen, S. \& Coulson, A. R. (1977). DNA sequencing with chain-terminating inhibitors. Proceedings of the National Academy of Sciences of the United States of America 74, 5463-5467.

SCHLEIFER, K. H. \& KILPPER-BÄLZ, R. (1987). Molecular and chemotaxonomic approaches to the classification of streptococci, enterococci and lactococci: a review. Systematic and Applied Microbiology 10, 1-19.

Shiroza, T., Ueda, S. \& Kuramitsu, H. K. (1987). Sequence analysis of the gtfB gene from Streptococcus mutans. Journal of Bacteriology $169,4263-4270$.

Silhavy, T. J., Berman, M. L. \& EnQuist, L. W. (1984). Experiments with Gene Fusions. Cold Spring Harbor, NY: Cold Spring Harbor Laboratory.

SMITH, D. J. \& TAUBMaN, M. A. (1978). Immunology and dental caries. In Textbook of Oral Biology, pp. 992-1006. Edited by J. H. Shaw, E. A. Sweeney, C. C. Cappuccino \& S. M. Meller. Philadelphia: W. B. Saunders.

Ueda, S., Shiroza, T. \& Kuramitsu, H. K. (1988). Sequence analysis of the $g t f C$ gene from Streptococcus mutans GS-5. Gene 69, 101-109.

W ALKer, G. J. \& JACQues, N. A. (1987). Polysaccharides of oral streptococci. In Sugar Transport and Metabolism in Gram-positive Bacteria, pp. 39-68. Edited by J. Reizer and A. Peterkofsky, Chichester: Ellis Horwood.

Wong, C., Hefta, S. A., Paxton, R. J., Shively, J. E. \& Mooser, G. (1990). Size and subdomain architecture of the glucan-binding domain of the sucrose :3- $\alpha$-D-glucosyltransferase from Streptococcus sobrinus. Infection and Immunity 58, 2165-2170.

YANNISCH-PERRON, C., VieIRA, J. \& Messing, J. (1985). Improved M13 cloning vectors and host strains: nucleotide sequences of the M13mp18 and pUC19 vectors. Gene 33, 103-119. 